\title{
The independence of private versus public abortion providers: implications for abortion stigma
}

\section{Anonymous}

Author's name and address withheld

Received 8 February 2012 Accepted 9 August 2012

\section{Background}

There is a live moral debate in the UK over the independence of specialised private and non-profit abortion clinics notably Marie Stopes and the British Pregnancy Advisory Service (BPAS) - that are licensed to provide state-funded abortion services, including pre-abortion counselling to discuss options and future contraceptive methods. ${ }^{1} \quad$ Currently, around $60 \%$ of state-funded abortions are outsourced and the rest are provided directly by the National Health Service (NHS). ${ }^{2}$ Conservative MP, Nadine Dorries, with the support of the antiabortion lobby, argues that outsourced clinics should not be allowed to provide pre-abortion counselling because they have a business interest in encouraging women to proceed with an abortion. Pro-choice advocates have hit back by defending the practices of Marie Stopes and BPAS. However, they have not considered the implications of the NHS filling the service gap if outsourced clinics are sidelined by an amendment to the Health and Social Care Bill. ${ }^{3}$ Although Nadine Dorries' initial amendment failed to make it into the legislation, the Government plans to introduce a second amendment after a consultation. ${ }^{4}$

In this opinion piece I seek to make the case that a larger role for the NHS in providing abortion services in the UK could exacerbate abortion stigma. There is a general belief amongst pro-choice advocates that abortion stigma is associated with the privatisation of abortion because it creates the perception that abortion is outside mainstream reproductive health programmes. ${ }^{5}$ From my own abortion experiences in both Australia and the UK, I believe that in countries where abortion is not legally available 'on-demand', abortion stigma will be higher in the public system versus the private system. This is because the public system must make value judgments in order to decide how to allocate limited resources. Therefore, society at large and Parliament (which is not formally separated from the Church in the UK) have complete moral agency over abortion services. This has implications for implementing the NHS constitution, which gives patients the right to access services without discrimination. ${ }^{6}$

\section{Personal experience}

I am 35-years-old and I have had four abortions. Two were the result of diaphragm failure in 15 years of use, and two were the result of having unprotected sex only twice during this period. I had three abortions in different private clinics in New South Wales (NSW), Australia, where the state (Medicare) does not cover the cost. Most recently I had a statefunded abortion in an NHS hospital in England, UK. Abortion has the same medicalised (as opposed to on-demand) legal status in NSW and England. Under British legislation (which excludes Northern Ireland), abortion is legal when two doctors agree that it is necessary to prevent undue physical or psychological harm to a pregnant woman. Under NSW legislation, only one doctor is required to make this assessment. ${ }^{7}$

Each time I had an abortion in NSW I met with the surgeon for pre-abortion counselling to discuss my life situation, contraceptive preferences and anxieties about pregnancy and abortion. Each time I felt informed, safe and respected. In the UK I never met the surgeon who performed the procedure and the preabortion counselling I received felt objectifying, threatening and stigmatising. The referring general practitioner and the hospital consultant both accused me of costing the NHS money because of my 
philosophical opposition to pharmaceutical (the pill) and invasive [(intrauterine device (IUD)] contraceptive methods. The consultant also threatened to not approve the abortion because I refused to have an IUD inserted during the procedure, despite my and my partner's assurances that we are now committed to only using condoms (in place of the diaphragm). She dismissed as unfounded my concerns about the risk of perforation, infection and ectopic pregnancy from an IUD. Contrary to Royal College of Obstetricians and Gynaecologists' guidelines, she told me that abortion is unsafe and having four abortions would be physically damaging. ${ }^{8}$ Neither doctor enquired about my psychological health (including my fear of being admitted to a large hospital crawling with germs, and my isolation from family and friends in Australia) but rather added to my anxiety by implying that in the future I may be forced to have an unwanted child, despite the fact that no technology is $100 \%$ effective.

\section{Abortion stigma and the NHS}

In part my UK experience might be explained by particular doctors not complying with their responsibilities under the NHS constitution. For example, Section 3b states: "You should aim to view the services you provide from the standpoint of a patient, and involve patients, their families and carers in the services you provide, working with them, their communities and other organisations, and making it clear who is responsible for their care". ${ }^{6}$ However, I believe that a more fundamental reason why the NHS is a conduit for abortion stigma is that it is not 'independent'. Indeed, because abortion is not a right in the UK, the morality of state-funded abortion is framed by members of parliament, including the 26 Church of England bishops and archbishops who are entitled to sit and vote in the House of Lords. ${ }^{9}$ Discrimination against women who do not conform to mainstream cultural and religious stereotypes is exemplified by the consent form I was asked to sign. The last section (reproduced below) was titled 'Funeral Arrangements'. It offered only two options and I was encouraged to select Option ' $a$ ' as the default:

(a) I ... (mother's name) consent to my baby/fetus that is obtained being taken for a shared cremation by the Department of Pastoral and Spiritual care (Chaplaincy).

(b) I wish to make my own arrangements for a private funeral or disposal of my baby/fetus.

The NHS consent form contributes to abortion stigma on three fronts. First, by substituting 'patient's name' with 'mother's name', it implies that the decision to have an abortion is comparable to killing one's own child rather than receiving reproductive health care. It is true that this interpretation of a fetus is consistent with the Royal College of Nursing guidance Sensitive Disposal of All Fetal Remains, which states that a fetus is comparable to a living person. ${ }^{10}$ However, it is not a universal interpretation and it sets women up to fail in the role of mother that has been imposed upon them simply because they have chosen to have an abortion. Second, it reduces 'womanhood' to 'motherhood' by invoking ceremonies that perpetuate the norm that all women need motherhood at all times so women who abort will naturally and perpetually mourn their loss. ${ }^{5}$ Finally, as an atheist I was cornered into contradicting my personal beliefs (Option a) in order to avoid the unwanted task of personally arranging for the disposal of a fetus (Option b). In NSW, private abortion providers can operate independently of broader cultural norms around motherhood and religious patronage. Disposal of an aborted fetus is therefore treated discretely like other unwanted pieces of tissue. This mitigates abortion stigma within the health system, despite abortion falling under the Crimes Act.

\section{Conclusions}

As a public health researcher, I hope that sharing my abortion experiences with the medical community can shed some light on the current debate over the independence of abortion services. Until abortion is defined as a right, we should be sceptical of antiabortionist attempts to decrease the private provision of abortion services because the combination of funding trade-offs and cultural and political power in the public system risks exacerbating abortion stigma. Abortion stigma is a form of discrimination that contradicts medical ethics and contributes to the death and injury of women around the world.

Competing interests None.

Provenance and peer review Not commissioned, externally peer reviewed.

\section{References}

1 Hansard. House of Commons debate. Wednesday 7 September 2011.

2 Department of Health. Abortion Statistics, England and Wales: 2009. Statistical Bulletin 2010/1. London, UK: Crown, 2010.

3 Curtis P, Quinn B. Abortion rules shakeup could set system back 25 years, says GPs' chief. The Guardian, 29 August 2011.

4 Simons N, Dorries N. MPs overwhelmingly reject abortion law amendment. The Huffington Post UK, 7 September 2011.

5 Kumar A, Hessini L, Mitchell EMH. Conceptualising abortion stigma. Cult Health Sexuality 2009;11:625-639.

6 National Health Service. The NHS Constitution for England. London, UK: Department of Health, 2012.

7 Drabsch T. Abortion and the Law in New South Wales (Briefing Paper No. 9/05). Sydney, Australia: NSW Parliamentary Library Research Service, 2005.

8 Royal College of Obstetricians and Gynaecologists (RCOG). The Care of Women Requesting Induced Abortion: Summary (Evidence-based Clinical Guideline Number 7). London, UK: RCOG, 2011.

9 Purvis M. House of Lords: Religious Representation. Lords Library Note 2011/036. London, UK: House of Lords, 2011.

10 Royal College of Nursing (RCN). Sensitive Disposal of All Fetal Remains: Guidance for Nurses and Midwives. London, UK: RCN, 2007. 